Christine R. Jenkins ${ }^{1,2,3}$

christine.jenkins@sydney.edu.au

\title{
Review
}

\section{Towards precision in defining COPD exacerbations}

COPD is the most prevalent chronic respiratory disease worldwide and a major cause of disability and death. Acute exacerbations of COPD remain a key feature of the disease in many patients and research assessing interventions to prevent and treat them requires a robust definition with high sensitivity and specificity. To date, no such definition exists, and multiple different definitions are used in clinical studies depending on the research question. The strengths and weaknesses of current definitions are discussed in the context of evolving knowledge and different settings in which studies are undertaken. Whether identification and recording of exacerbations remains essentially clinical, or can be identified with a dependable biomarker, it should be sensitive and adaptable to context while retaining clarity and facilitating data collection. This is essential to progress a better understanding of the pathophysiology and phenotypic expression of exacerbations to reduce their impact and personal burden for patients.

\section{Introduction}

The global mortality and morbidity of COPD is high and has not changed appreciably, unlike many other noncommunicable diseases, over the past 20 years. The Global Burden of Disease Study reported that an estimated 545 million people worldwide had a chronic respiratory disease in 2017, equivalent to a $40 \%$ increase compared with the number of individuals affected in 1990, an increase disproportionate to global population growth [1]. COPD, with an overall global prevalence of $6 \%$, remained the most prevalent chronic respiratory disease worldwide and made up 55\% of chronic respiratory disease prevalence among men and women. This represents an increase in overall prevalence of $6 \%$, with a marked increase in women over the same period [2, 3]. Chronic respiratory disease is almost equally prevalent in high income countries and low-middle income countries (LMICs), although with vastly different aetiologies and potentially still serious underdiagnosis in LMICs. Chronic respiratory diseases account for $7 \%$ of total all-cause deaths globally, and in 2017 were the third leading cause of death, close behind cardiovascular disease and cancer.

COPD, as the major portion of this high prevalence, also confers very high morbidity [4]. Disability (disability-adjusted life years (DALYs)) due to chronic respiratory diseases increased 13\% between 1990 and 2017, and COPD accounted for the lion's share of this [1]. Some gains have been made, the prevalence, mortality, and DALY rates per 100000 people dropping significantly
Cite as: Jenkins CR. Towards precision in defining COPD exacerbations. Breathe 2021; 17: 210081. 
between 1990 and 2017 when adjusted for population growth and ageing. However, COPD remains a major global health issue, with increasing numbers of people affected and several of its major causes still not addressed in many regions of the world. This is despite the fact that much of the burden of COPD can be prevented by primary and secondary interventions ranging from public health and government policy to health infrastructure, skilled workforce development and community health literacy [5]. Even in high income countries, treatment-related costs consume a major portion of healthcare budgets [6, 7], and a major portion of these is the cost of exacerbations [7, 8]. Indirect costs of work and productivity loss are also substantial and just as great a burden for the patient as for wider society [6].

\section{Exacerbations of COPD}

Although COPD is a progressive illness marked by daily symptoms and in some patients, a slow decline in lung function and quality of life [9, 10], acute exacerbations of COPD remain a key feature of the disease in many patients. Exacerbations are more likely to occur in patients with a high symptom burden, and in themselves add further burden of disease and impairment of health status [9], as well as risk of more frequent severe events [11-13]. Patients describe exacerbations as having a major impact on their quality of life [9]. Unscheduled visits to healthcare, whether primary, secondary or tertiary, are costly $[14,15]$, disruptive and most importantly to the patient, moments of extreme physical [6] and emotional stress [16, 17], and for some, life-threatening [18-20].

For the purposes of this introductory discussion, I will define exacerbations of COPD clinically, as episodes of acute worsening of symptoms, leading to additional therapy and hospitalisations, that punctuate a patient's journey more frequently as lung function and ventilatory impairment worsen with time. However, as will become clear, within and between patients with COPD, exacerbations are heterogeneous events, with multiple causes, different expression, and varying impact and clinical responses. A satisfactory definition is not only a semantic challenge, but also an epistemological and environmental one. Most definitions of COPD exacerbations contain both a symptom-based component and an event-based component. There are inadequacies with an emphasis on either of these approaches [21], although alternative definitions have not proven to be superior as a general rule, even if fit for specific purposes, such as clinical trials [22], database analysis [23] or studies examining pathophysiology.

Exacerbations vary in frequency in individual patients and most patients do not exacerbate at exactly the same rate year after year [24, 25]. In many studies [26], including the Evaluation of
COPD Longitudinally to Identify Predictive Surrogate End-points (ECLIPSE) [27] and the Subpopulations and Intermediate Outcome Measures in COPD Study (SPIROMICS) [28], a significant proportion of so-called frequent exacerbators or infrequent exacerbators change status over a 3-year period, indicating that propensity to exacerbation is not a fixed property and either naturally or through both pharmacological $[29,30]$ and nonpharmacological [31] interventions, patients can change their exacerbation status.

Exacerbation frequency is strongly related to severity of airflow limitation as measured by forced expiratory volume in $1 \mathrm{~s}\left(\mathrm{FEV}_{1}\right)$. This was demonstrated in a recent study from Norway, where the incidence rate ratios for utilisationdefined acute exacerbations of COPD were 2.45 (95\% Cl 1.22-4.95), 3.43 (95\% Cl 1.59-7.38), and 5.67 (95\% Cl 2.58-12.48) with Global Initiative for Obstructive Lung Disease (GOLD) spirometric stages II, III, and IV, respectively [32]. Similarly in an analysis of the Copenhagen General Population Study [23], compared to individuals with GOLD 1, the risk of exacerbations was 17-fold for GOLD 4, five-fold for GOLD 3, and two-fold for GOLD 2.

At a population level, being more prone to exacerbations is associated with being older, having worse $\mathrm{FEV}_{1}$, lower levels of daily physical activity, more hours of sedentary time in a day, a greater number of comorbidities, higher dyspnoea scores and higher probability of anxiety and depression [25]. In the SPIROMICS population, having a consistent acute exacerbation profile ( $\geq 1$ event per year for 3 years) was associated with higher baseline symptom burden, previous exacerbations and greater evidence of small airway abnormality on computed tomography (CT), compared with having no acute exacerbations [28].

Many investigators have developed tools to enable practitioners to estimate the risk of developing an exacerbation, most recently the ASSESS tool [33]. In a study using the ECLIPSE 3-year observational study to validate the ASSESS findings, predicted exacerbation and observed exacerbation rates were similar (1.31 versus 1.20 events per year for all exacerbations and 0.25 versus 0.27 events per year for severe exacerbations). Area under the curve (AUC) was 0.73 (95\% Cl 0.70-0.76) for two or more exacerbations and $0.74(95 \% \mathrm{Cl} 0.70-0.78)$ for at least one severe exacerbation. At an individual level, many factors increase exacerbation risk, particularly increasing age, worse $\mathrm{FEV}_{1}$, having a history of exacerbations, a higher modified Medical Research Council (mMRC) dyspnoea score [34], worse health status measured by COPD Assessment Test (CAT) or St George's Respiratory Questionnaire (SGRQ), having gastro-oesophageal reflux [35] and a chronic bronchitis phenotype [9, 36].

The economic costs of COPD associated with exacerbations [8, 37] are substantial. However, the arguments for using exacerbations as an endpoint extend beyond their economic impact, even 
though this has proven to be a vital aspect of the regulatory process and approvals for drug availability and affordability in global markets. The strongest arguments for a robust definition primarily derive from the very severe consequences of exacerbations for patients [18, 20, 38].

\section{A brief history}

In early clinical trials of COPD, many different clinical and physiological characteristics were studied, but in particular, measures of airway obstruction. Despite the reproducibility of the $\mathrm{FEV}_{1}$ and its relationship to other important clinical outcomes in patients with COPD, it is not a sensitive measure of change during acute exacerbations, can be difficult to perform when patients are unwell, and does not relate strongly to the symptom burden and impaired quality of life that characterises acute exacerbations of COPD.

However, in 1987, significant progress was made when ANTHONISEN et al. [22] used a graded definition of exacerbations in a trial of antibiotics for COPD exacerbations. This definition contained objective criteria that could be used in other studies and enabled some quantification of individual components. These exacerbations were described by major and minor symptoms and somewhat arbitrarily graded by type. Type 1 was characterised by increased dyspnoea, sputum volume and sputum purulence; type 2 when two of these symptoms were present; and type 3 when one of the three major symptoms was present in addition to at least one of either an upper respiratory infection within the past 5 days, fever without other cause, increased wheezing or cough, or an increase in respiratory rate or heart rate by $20 \%$ as compared with baseline. Despite the appeal of these objective features, and their subsequent use in many clinical studies, they were not derived from a database or case series describing individual characteristics or patterns of exacerbation. They describe symptoms, but do not relate to the underlying pathophysiology or the likely evolution of the exacerbation.

Exacerbations were still variably described in the literature and in small clinical trials prior to a definition proposed in 2000 by RodRIGUEzRoIsIN [39] in a paper summarising an expert workshop that reached a consensus definition for use in trials and studies. This definition describes an exacerbation as " a sustained worsening of the patient's condition from the stable state and beyond normal day to day variations, that is acute in onset and necessitates change in regular medication in a patient with underlying COPD" [39]. This was a pivotal moment in defining exacerbations, as it introduced a standardised definition for counting exacerbations, was a realistic definition for clinical trials and aligned with clinical care. Although the requirement for symptom worsening limits its use for identifying exacerbations in databases and routinely collected healthcare data, the inclusion of medication change could be used as a surrogate marker if associated with a healthcare visit.

Subsequent to this, many studies incorporated versions of these symptom descriptors, particularly emphasising worsening of respiratory symptoms that required treatment with oral corticosteroids or antibiotics or both, as judged by the attending clinician. Dyspnoea became the major, dominant symptom, based on evidence from studies that clearly highlighted its importance to patients and as a trigger for initiating greater intensity of treatment and seeking medical care.

The rationale behind each component of the definition by RODRIGUEZ-ROISIN [39] is explained in the paper, which succinctly summarises the subsequent challenges. 20 years of experience has made investigators, epidemiologists and clinicians well aware of its strengths and weaknesses, and it is now worth revisiting this definition, and looking at potential refinements that would add to its utility while not reducing its capacity to be applied in many different contexts. I will address each of these in the light of the experience gained by investigators as they implemented it in many different clinical trials, observational studies and database analyses.

\section{Dissecting the definition}

\section{Sustained worsening from the stable state}

RodRIGUEZ-RoIsIN [39] suggests "The terminology, worsening of the patient's condition, is relatively imprecise because of the absence of established clinical markers, signs, or symptoms".

\section{Sustained worsening of the stable state}

Most patients find it difficult to identify the very early stages of an exacerbation and to distinguish their increased symptoms from day-to-day variability. This has several immediate consequences for patients, regarding commencing treatment [40] and achieving the best possible outcomes [41]. Underrecognition of exacerbations is a concern [41, 42], in that these events may lead to worse outcomes, specifically longer duration, poorer quality of life and increased risk of hospitalisation [43, 44], but over-reporting can also occur (events that are identified by patients as an exacerbation but are not accompanied by symptom change in diary cards [44], or do not result in healthcare contact or change of treatment [42]). In most studies, unreported exacerbations have less intense and often fewer symptoms [43] and appear to be milder events [40] even though they have a cumulative impact on health status $[41,44]$. Whether in a clinical trial or through routinely collected data, worsening of the stable state is limited by its dependence on patient recognition, which it is well established can result in both over- [45] and 
undercounting [46]. Compared with reported exacerbations, unreported exacerbations usually show a lower median number of key symptoms, less worsening for each key symptom, and a significantly lower proportion of exacerbations with two and three key symptoms. Conversely, more unreported exacerbations (83.3\%) than reported exacerbations (28.8\%) exhibited a single worsening symptom.

With regard to the absence of established clinical markers, signs, or symptoms, it is an unfortunate fact that we are still struggling to develop, test and embed these in clinical and research practice. We know the specific symptoms most often associated with an exacerbation, but we do not have an adequate scoring system to rank these, even though we have several validated tools focused on symptoms that enable us to better understand the impact of COPD in patients' lives, particularly the CAT and the SGRQ Both are highly useful for assessments of change over time from an exacerbation, but they were not designed to detect and measure exacerbations in themselves [47]. Tools to detect and measure the onset, severity and resolution of an acute deterioration have proven challenging to develop.

What are the key symptoms of a COPD exacerbation? ANTHONISEN et al. [22]. carefully divided exacerbation symptoms into major and minor, particularly focused on achieving a definition suitable for a clinical trial of antibiotics. A similar categorisation has been useful in other analyses, but it is crucial to acknowledge that patients symptoms [48], their perceptions [49] and ability to grade these vary markedly. Cultural [49], agerelated and gender-based $[36,50,51]$ differences also influence this, as does severity of COPD [34, 52]. Patient-reported outcome (PRO) measures vary greatly in sensitivity and specificity for identification of exacerbations in patients with COPD [53,54], and specific definitions [55] and tools for this purpose must be carefully chosen $[56,57]$.

Dyspnoea is a major symptom of COPD and intensifies during exacerbations [46, 58]. Most patients recognise it as a clear indication that they are more unwell [40]. Cough and sputum, and chest symptoms are also key symptoms and with dyspnoea account for most of the quantitative change from the stable state during an exacerbation $[49,59]$. Many other symptoms are highly variable, dependent on underlying aetiology of the exacerbation, clinical disease features, whether the patient has a chronic bronchitic or emphysematous phenotype, the presence of comorbidities and the cultural setting of the patient and their carers. Systemic features such as marked fatigue may be prominent but lack specificity and so the respiratory symptoms are essential to identify worsening of the patient's condition as due to a COPD exacerbation.

\section{Severity}

Severity is another aspect of worsening: how much worse? Exacerbation grading is variably defined in clinical studies and trials. The grading most commonly accepted or used in studies involves an event-based assessment for moderate-severe exacerbations. In randomised controlled trials (RCTs) exacerbations are often classified as mild if they are treated with short-acting bronchodilators only; moderate if treated with short-acting bronchodilators plus antibiotics and/or oral corticosteroids; and severe if the patient visits the emergency room (ER) or requires hospitalisation because of an exacerbation [60]. In some RCTs, exacerbations resulting in admission to intensive care or death are categorised as very severe [61]. These definitions have varied considerably in clinical trials, often depending on the intervention and its anticipated primary effect [62]. In many RCTs mild exacerbations are defined on symptoms alone, particularly an increase in, or new onset of, cough, sputum, dyspnoea, wheezing or chest tightness [63].

Other definitions of exacerbation severity used in RCTs have included any inhaled medication increases in mild exacerbations, systemic corticosteroids only in moderate exacerbations $[62,64]$, and in the severe category, only hospital admissions or deaths [65]. The inclusion of ER presentations in the severe category depends on geography [66], access and affordability of care and in many settings does not indicate a severe exacerbation on clinical grounds. It may thus overestimate the prevalence of severe exacerbations. In some high income countries, patients requiring admission based on clinical severity form only a small proportion of all those with exacerbations $[18,32]$, whereas in other settings admissions are those most commonly recorded in a trial, and many community based events go unrecorded [67]. It is also important to note that in many countries, self-management of exacerbations is based on a written action plan [68] and a patient's own supply of antibiotics or corticosteroids, which may also result in under-reporting if the patient fails to recall the event or does not seek medical assistance at the time $[54,69]$. Some RCTs of COPD therapies have required sustained symptom changes that may be purposeful in terms of the intervention [ 58 , $70,71]$, but can also result in under-recognition if prespecified criteria are not met $[62,71]$.

The absence of established clinical markers

Biomarkers have the potential to identify COPD exacerbations in the real world for patients and clinicians, and in clinical trials. An ideal exacerbation biomarker would be an accessible, reproducible, externally validated objective measure that either signals an exacerbation or confirms one, when associated with symptoms. Such a biomarker would help to eliminate the subjectivity of symptom worsening beyond day-to-day variability, but no such marker has yet been identified, despite major investment in cohort studies and clinical trials. 
Many investigators have tried to identify a blood test that may be an effective biomarker for COPD exacerbations. The prime contenders, based on current evidence would be C-reactive protein (CRP) or fibrinogen, as raised levels are associated with a greater probability of exacerbating. One problem with both biomarkers in COPD is that an elevation may be a continuous, almost steady state for some patients [72]. In the ECLIPSE study, inflammatory biomarkers in peripheral blood were quantified in 1755 COPD patients followed over 3 years. At baseline, $30 \%$ of COPD patients did not show evidence of systemic inflammation whereas 16\% had persistent systemic inflammation evidenced by elevations in the assessed markers [72]. A high white cell count has been associated repeatedly with some exacerbations and exacerbation propensity, but is a nonspecific finding, indicative of many types of infection and likely to rise if patients have already commenced systemic steroids. High blood neutrophil counts were also associated with a frequent exacerbation phenotype and mortality in ECLIPSE [35, 73]. Similarly, elevation of blood eosinophils $\geq 3 \%$ or $\geq 300$ cells $\cdot \mathrm{mm}^{-3}$ appears to identify a subpopulation of patients at higher risk of exacerbations and a greater probability of a positive benefit from short-course corticosteroids, but this feature varies and may be evident when clinically stable and during an exacerbation. Eosinophilia may also indicate more benefit in exacerbation reduction from inhaled corticosteroids [74], but background counts or those taken at exacerbation do not have any rule-in or rule-out utility. Given this is a long term (albeit fluctuating) phenotypic marker, it lacks sensitivity and specificity for identifying exacerbations.

Biomarker repeatability was assessed in a subset of patients with COPD, ex-smoker controls with normal lung function and healthy nonsmokers selected from the ECLIPSE cohort, at baseline and 3 months, with CRP showing wide variability [75]. Fibrinogen was the most repeatable biomarker and weakly correlated with 6-min walking distance, exacerbation rate, BODE (body mass index, airflow obstruction, dyspnoea, exercise capacity) index and MRC dyspnoea score. Several inflammatory markers appear to reflect longer term disease activity: CRP, fibrinogen, interleukin- 6 and surfactant protein-D were significantly elevated in subjects with exacerbations within 30 days of the 3-month visit compared with those individuals that did not exacerbate [72]. Persistence of a high CRP in ECLIPSE was a flag for predisposition to recurrence and overall higher exacerbation frequency, but did not have specificity for accurate timing [76]. In other studies, lower interleukin-15 concentrations, and higher interleukin-8 concentrations were associated with higher probability of having $>1$ exacerbation per year. However, none of these biomarkers have proven to be useful for contemporaneous confirmation of an exacerbation.
The definition acknowledges that a patient's stable state may fluctuate, and therefore includes the wording, beyond normal day-to-day variations

The usefulness of daily diaries in identifying exacerbations has been well demonstrated by the evidence that has emerged from the East London cohort and others like it. This is a prospective longitudinal cohort of COPD patients recruited from outpatient clinics of the London Chest and Royal Free Hospitals, London, UK and followed for a minimum of 2 years. Daily measures of symptoms, medication use, hospitalisations and clinic visits were recorded by each participant, including symptom increases above baseline, enabling identification of COPD exacerbations. These cohorts have yielded exceptionally valuable information about symptoms, frequency of flare-ups, healthcare contact, management and outcomes. It is however crucial to appreciate that daily record keeping over months to years is arduous and those who do it well may be a subset and not necessarily representative of the broader COPD population. The development of PRO tools have enabled standardisation of these measures and have been a vital addition to understanding the pattern and impact of exacerbations at an individual level [56], as well as facilitating comparisons across different demographic groups.

Electronic diaries and smartphone-based applications that enable text entry, responses to questions and uploading of physiological data such as heart rate, respiratory rate, airflow measurements and oximetry are an inevitable development from such diaries. Automatically collected data from nanodevices and microchips have opened up a raft of possibilities for self-monitoring, home care, outreach services and early discharge [46].

Electronic diaries have the benefit of real time data entry and upload, eliminating entries based on recall and helping to prevent fictional entries, often by automatically entering a time and date stamped measurement (such as peak flow). Several PROs have been automated and embedded in electronic diaries [77], recording symptom frequency and severity of daily COPD symptoms. These diaries may also have software that computes day-to-day variability and signals to patients when symptom scores or peak flow rates deviate by more than a predetermined margin from normal. Tools, such as the Exacerbations of COPD Tool (EXACT) questionnaire $[48,78]$, can be integrated into smartphones, providing a validated, sensitive questionnaire that enables acute symptom changes to be recorded for the detection of exacerbations in clinical trials.

\section{A sustained worsening that is acute in onset}

A key feature of the definition provided by RodRIGUez-RoIsIn [39] is that the exacerbation 
is defined by being acute in onset. This is also stated in the current definition as it appears in the Global Strategy for the Diagnosis, Management, and Prevention of Chronic Obstructive Pulmonary Disease: "An exacerbation of COPD is defined as an acute worsening of respiratory symptoms that results in additional therapy" [79]. However, there is now good evidence from a variety of sources $[38,80]$ that exacerbations may be of a gradual onset and this should be reflected in the definition.

In a careful and highly informative analysis of the East London COPD cohort data, AARON et al. [80] defined an exacerbation commencement date as the first of two or more consecutive days on which the patient recorded two or more new or worsening symptoms, at least one of which was a major symptom, 55\% of these resolved spontaneously. Amongst those who developed an exacerbation, the onset was sudden in $56 \%$ and the exacerbation threshold was crossed on the same day symptoms began. By contrast, 44\% of exacerbations were characterised by gradual onset of symptoms with a median duration from symptom onset to exacerbation of 4 days. Patients who experienced sudden onset exacerbations had greater mean daily symptom scores, greater peak symptom scores, earlier peak symptoms and shorter median recovery times back to baseline health status. Gradual onset exacerbations were statistically associated with a longer duration of exacerbation recovery (OR 1.28, 95\% Cl 1.061.54, $\mathrm{p}<0.010)$. Others have also shown that COPD exacerbations may exhibit a gradual onset, and these may also be more likely to be slower to resolve.

Further, several studies suggest that the particular symptom thresholds and definitions within a symptomatic event clearly influence the identification of those events [44, 81]. Establishing a low threshold, however, has risks and may distort treatment effects. These risks include over-sensitivity and the inclusion of small changes in symptoms that are part of day-to-day variability, or milder exacerbations that may be shorter-lived or have only a small impact [46]. In some study designs, low treatment thresholds may have been deliberately included to enable a primary end-point to be reached more rapidly, or an intervention to appear to have greater efficacy in reducing exacerbations. However, the nature of the treatment that is changed is also important in counting exacerbations. Some clinical trials have defined an exacerbation as an event in which bronchodilators and/or inhaled medications are increased, and there are different impacts depending on which these are. Medications such as short-acting $\beta_{2}$-agonists treat the symptoms, primarily dyspnoea, whereas others, such as inhaled corticosteroids, can address the underlying mechanisms in some patients and may reverse the exacerbation.

\section{The definition requires that the change in condition necessitates a change in regular medication}

As already noted, a problem with symptom or treatment defined COPD exacerbations, is what constitutes a change in regular medication. Not only does the word "change" matter, but also regular medication [82]. This is more of an issue than it used to be because patients across the world are taking very different medications as their regular medications [32]. In medium-high income countries, patients may be prescribed best practice medications based on current guidelines and a well-resourced healthcare system and affordable medications [79]. For some patients in high income settings and most in low income countries, their regular medications are shortacting $\beta_{2}$-agonists and antimuscarinics alone $[83,84]$.

Most commonly, prescription of systemic corticosteroids or antibiotics signifies the recognition of an exacerbation by the health professional or the patient. These two players, the patient and the health professional involved in exacerbation identification can have very different perspectives [85, 86], and may alter treatments for different reasons [40, 49]. In $\mathrm{RCTs}$, the randomisation may be trusted to distribute this variability evenly between the intervention arms, but the problem of identifying reasons for treatment change, and sometimes the true severity of the event remains. It is now well known that in winter, the prescription of antibiotics during exacerbations is more common [34, 87], but this may be empiric and does not necessarily indicate careful clinical assessment for a bacterial aetiology.

Local access to healthcare services and affordability of care are immensely important in determining whether a moderate-severe exacerbation is recorded. People living in regional and remote communities, even in high income countries [66], often have much more significant difficulty accessing timely medical care, more hospital admissions and longer length of stay. People in low income countries may not have access to short-acting inhaled medications unless they present to hospital [84, 88]. Given the burden of COPD falls disproportionately on these communities, there is a need to address this deficiency in the definition of COPD exacerbations in order to accurately estimate the burden of COPD and the urgent need for preventative measures to reduce this. Health literacy, poverty, geographical access to care and affordability of medications all influence the measurement of exacerbations in socioeconomically deprived areas, whether an event-based or symptom/treatment-based definition is used. The mechanisms for the development of COPD in low income settings result from exposure to a range of noxious agents and inhalations that extend far beyond tobacco $[89,90]$, and along with early life respiratory insults [84] may also contribute to failure to achieve normal lung growth in early adult life $[90,91]$. Symptom 
worsening and treatment response may be different in these diverse aetiologies of COPD and much more research is needed to fully explore this.

\section{What do we need for the future?}

In summary, the definition of a COPD exacerbation proposed in 2000 has served well as a template for many carefully conducted RCTs, clinical cohort studies and database analyses. Each of these purposes has necessitated slightly modified versions of the definition, based on the rationale for the study and the data collected. This has led to a plethora of exacerbation definitions which although only superficially different, can have very marked repercussions for estimations of burden of COPD and the effects of treatment.

It is essential that we acknowledge the imprecise aspects of our different exacerbation definitions, particularly as healthcare is changing so rapidly and events previously associated with most severe exacerbations, such as hospitalisations, are much less common. Other contemporary influences may lead to changes in our capacity to record the true burden of exacerbations. As an example, the trend to phenotype patients and their exacerbations for targeted treatment approaches, would result in fewer recorded events if guidelines advise against systemic corticosteroids for an exacerbating patient with low eosinophils. Administrative pressures to reduce admissions, the increase in patient self-management capacity and the wider availability of home-based care through clinical outreach services must be considered as changes that could also influence the recording of severe exacerbations if the commonly used definitions in large RCTs are retained.

We focus on exacerbations because of their impact on patients and healthcare systems. In the past 20 years, billions of dollars have been spent conducting RCTs to assess the efficacy of medications and interventions to reduce their frequency and severity, with mean reductions of $\sim 20 \%$ through optimal treatment regimens in highly selected patients, but there is a persistent residual exacerbation rate which remains a major burden. I have not addressed the aetiology of COPD exacerbations in this review, but the experience of the coronavirus disease 2019 (COVID-19) pandemic has demonstrated that social distancing, masking and hand hygiene have had a major impact in reducing exacerbations [92] and hospital admissions [93], an observation that has been made around the world in vastly different settings [94]. We must make immediate use of this information for the good of our patients and clinical trials with careful counting of exacerbations are probably not needed to demonstrate the benefits of avoiding exposure to respiratory viruses.

It is likely that the different definitions of a COPD exacerbation will evolve as the search for a standardised measure continues, and probable that there will never be a single definition that can meet all purposes. Whether identification and recording of exacerbations remains essentially clinical, or can be identified with a dependable biomarker, it should be sensitive and adaptable to context while retaining clarity and facilitating data collection. In every study, investigators should ensure that variations on the commonly accepted definition are made explicit and entirely justified. Research is urgently needed to explore the use of definitions in low income countries to enable a better understanding of the impact of the causes, impact and management of COPD exacerbations in circumstances dramatically different to middle to high income countries where the majority of large COPD trials are undertaken. Finally, we have much more to do to progress a better understanding of the pathophysiology, aetiology and phenotypic expression of exacerbations to reduce their impact and personal burden for patients.

\section{Affiliations}

\section{Christine R. Jenkins ${ }^{1,2,3}$}

${ }^{1}$ Respiratory Group, The George Institute for Global Health, Sydney, Australia. ${ }^{2}$ UNSW Sydney, Sydney, Australia. ${ }^{3}$ Concord Clinical School, University of Sydney, Sydney, Australia.

\section{Conflict of interest}

C.R. Jenkins reports receiving a research grant, paid to their institution, from GlaxoSmithKline, outside the submitted work. Payment or honoraria for lectures, presentations, speaker bureaus, manuscript writing or educational events from Boehringer Ingelheim, GlaxoSmithKline, AstraZeneca, Novartis and Sanofi-Genzyme (member of local and global advisory boards for asthma and COPD, speaker at online/video conferences/ educational events 2019-2021), outside the submitted work. Payment for expert testimony from Novartis (advice to Therapeutics Goods Authority Australia), outside the submitted work. Support for attending meetings and/or travel from Boehringer Ingelheim, AstraZeneca, Novartis and Sanofi-Genzyme (for global advisory boards 2019), outside the submitted work. Participation on a Data Safety Monitoring Board or Advisory Board for an AstraZeneca funded study, independent DSMB, outside the submitted work. Leadership or fiduciary role in other board, society, committee or advocacy group, paid or unpaid for the Lung Foundation Australia, outside the submitted work. 


\section{References}

1. GBD Chronic Respiratory Disease Collaborators. Prevalence and attributable health burden of chronic respiratory diseases, 1990-2017: a systematic analysis for the Global Burden of Disease Study 2017. Lancet Respir Med 2020; 8: 585-596.

2. Gershon A, Hwee J, Victor JC, et al. Mortality trends in women and men with COPD in Ontario, Canada, 1996-2012. Thorax 2015; 70: 121-126.

3. Vos T, Flaxman AD, Naghavi M, et al. Years lived with disability (YLDs) for 1160 sequelae of 289 diseases and injuries 19902010: a systematic analysis for the Global Burden of Disease Study 2010. Lancet 2012; 380: 2163-2196.

4. McGrath R, Al Snih S, Markides K, et al. The burden of health conditions for middle-aged and older adults in the United States: disability-adjusted life years. BMC Geriatr 2019; 19 : 100.

5. Burney P, Patel J, Minelli C, et al. Prevalence and populationattributable risk for chronic airflow obstruction in a large multinational study. Am J Respir Crit Care Med 2020; in press [https://doi.org/10.1164/rccm.202005-19900C].

6. Foo J, Landis SH, Maskell J, et al. Continuing to confront COPD international patient survey: economic impact of COPD in 12 countries. PLoS One 2016; 11: e0152618.

7. Dang-Tan T, Ismaila A, Zhang S, et al. Clinical, humanistic, and economic burden of chronic obstructive pulmonary disease (COPD) in Canada: a systematic review. BMC Res Notes 2015; 8: 464.

8. Dalal AA, Shah M, D'Souza AO, et al. Costs of inpatient and emergency department care for chronic obstructive pulmonary disease in an elderly Medicare population. J Med Econ 2010; 13: 591-598.

9. Hurst JR, Skolnik N, Hansen GJ, et al. Understanding the impact of chronic obstructive pulmonary disease exacerbations on patient health and quality of life. Eur J Intern Med 2020; 73 $1-6$

10. Jones PW, Anderson JA, Calverley PMA, et al. Health status in the TORCH study of COPD: treatment efficacy and other determinants of change. Respir Res 2011; 12: 71.

11. Hoogendoorn M, Hoogenveen RT, Rutten-van Molken MP et al. Case fatality of COPD exacerbations: a meta-analysis and statistical modelling approach. Eur Respir J 2011; 37 508-515.

12. Reilev M, Pottegard A, Lykkegaard J, et al. Increased risk of major adverse cardiac events following the onset of acute exacerbations of COPD. Respirology 2019; 24: 1183-1190.

13. Suissa S, Dell'Aniello S, Ernst P. Long-term natural history of chronic obstructive pulmonary disease: severe exacerbations and mortality. Thorax 2012; 67: 957-963.

14. Wallace $A E$, Kaila $S$, Bayer $V$, et al. Health care resource utilization and exacerbation rates in patients with COPD stratified by disease severity in a commercially insured population. J Manag Care Spec Pharm 2019; 25: 205-217.

15. Milewska A, Rysiak E, Zareba I, et al. Costs of treatment of chronic obstructive pulmonary disease. Adv Exp Med Biol 2016; 885: 67-75

16. Laurin C, Moullec G, Bacon SL, et al. The impact of psychological distress on exacerbation rates in COPD. Ther Adv Respir Dis 2011; 5: 3-18.

17. Montserrat-Capdevila J, Godoy P, Marsal JR, et al. Overview of the impact of depression and anxiety in chronic obstructive pulmonary disease. Lung 2017; 195: 77-85.

18. Colak Y, Afzal S, Marott JL, et al. Prognosis of COPD depends on severity of exacerbation history: a population-based analysis Respir Med 2019; 155: 141-147.

19. Almagro $P$, Calbo E, Ochoa de Echagüen A, et al. Mortality after hospitalization for COPD. Chest 2002; 121: 1441-1448.

20. Schmidt SAJ, Johansen MB, Olsen $M$, et al. The impact of exacerbation frequency on mortality following acute exacerbations of COPD: a registry-based cohort study. BMJ Open 2014; 4: e006720.

21. Sapey E, Bafadhel M, Bolton CE, et al. Building toolkits for COPD exacerbations: lessons from the past and present. Thorax 2019; 74: 898-905.
22. Anthonisen NR, Manfreda J, Warren CP, et al. Antibiotic therapy in exacerbations of chronic obstructive pulmonary disease. Ann Intern Med 1987; 106: 196-204.

23. Ingebrigtsen TS, Marott JL, Lange $P$, et al. Medically treated exacerbations in COPD by GOLD 1-4: a valid, robust, and seemingly low-biased definition. Respir Med 2015; 109: 1562-1568.

24. Rothnie KJ, Mullerova $\mathrm{H}$, Smeeth L, et al. Natural history of chronic obstructive pulmonary disease exacerbations in a general practice-based population with chronic obstructive pulmonary disease. Am J Respir Crit Care Med 2018; 198: 464-471

25. Mullerova $H$, Shukla A, Hawkins A, et al. Risk factors for acute exacerbations of COPD in a primary care population: a retrospective observational cohort study. BMJ Open 2014; 4: e006171.

26. Kardos $\mathrm{P}$, Vogelmeier $\mathrm{C}$, Worth $\mathrm{H}$, et al. A two-year evaluation of the 'real life' impact of COPD on patients in Germany: the DACCORD observational study. Respir Med 2017; 124: 57-64.

27. Donaldson GC, Mullerova $\mathrm{H}$, Locantore $\mathrm{N}$, et al. Factors associated with change in exacerbation frequency in COPD. Respir Res 2013; 14: 79

28. Han MK, Quibrera PM, Carretta EE, et al. Frequency of exacerbations in patients with chronic obstructive pulmonary disease: an analysis of the SPIROMICS cohort. Lancet Respir Med 2017; 5: 619-626.

29. Wing K, Williamson E, Carpenter JR, et al. Real world effects of COPD medications: a cohort study with validation against results from randomised controlled trials. Eur Respir J 2021 57: 2001586

30. Lipson DA, Barnhart F, Brealey N, et al. Once-daily singleinhaler triple versus dual therapy in patients with COPD. N EnglJ Med 2018; 378: 1671-1680

31. Liang J, Abramson MJ, Russell G, et al. Interdisciplinary COPD intervention in primary care: a cluster randomised controlled trial. Eur Respir J 2019; 53: 1801530.

32. Erdal M, Johannessen A, Eagan TM, et al. Incidence of utilization- and symptom-defined COPD exacerbations in hospital- and population-recruited patients. Int J Chron Obstruct Pulmon Dis 2016; 11: 2099-2108.

33. Adibi A, Sin DD, Safari A, et al. The acute COPD exacerbation prediction tool (ACCEPT): a modelling study. Lancet Respir Med 2020; 8: 1013-1021.

34. Jenkins C, Celli B, Anderson J, et al. Seasonality and determinants of moderate and severe COPD exacerbations in the TORCH study. Eur Respir J 2012; 39: 38-45.

35. Hurst JR, Vestbo J, Anzueto A, et al. Susceptibility to exacerbation in chronic obstructive pulmonary disease. $N$ Engl J Med 2010; 363: 1128-1138.

36. Sundh J, Johansson G, Larsson K, et al. The phenotype of concurrent chronic bronchitis and frequent exacerbations in patients with severe COPD attending Swedish secondary care units. Int J Chron Obstruct Pulmon Dis 2015; 10: 2327-2334.

37. Gershon AS, Guan J, Victor JC, et al. Quantifying health services use for chronic obstructive pulmonary disease. Am J Respir Crit Care Med 2013; 187: 596-601.

38. Kessler R, Stahl E, Vogelmeier C, et al. Patient understanding, detection, and experience of COPD exacerbations: an observational, interview-based study. Chest 2006; 130: 133-142.

39. Rodriguez-Roisin R. Toward a consensus definition for COPD exacerbations. Chest 2000; 117: Suppl. 2, 398S-401S.

40. Vijayasaratha K, Stockley RA. Reported and unreported exacerbations of COPD: analysis by diary cards. Chest 2008: 133: 34-41

41. Xu W, Collet JP, Shapiro S, et al. Negative impacts of unreported COPD exacerbations on health-related quality of life at 1 year. Eur Respir J 2010; 35: 1022-1030.

42. Frent SM, Chapman KR, Larbig M, et al. Capturing exacerbations of chronic obstructive pulmonary disease with EXACT. A subanalysis of FLAME. Am J Respir Crit Care Med 2019; 199: 43-51. 
43. Wilkinson TMA, Donaldson GC, Hurst JR, et al. Early therapy improves outcomes of exacerbations of chronic obstructive pulmonary disease. Am J Respir Crit Care Med 2004; 169: 1298-1303.

44. Langsetmo L, Platt RW, Ernst P, et al. Underreporting exacerbation of chronic obstructive pulmonary disease in a longitudinal cohort. Am J Respir Crit Care Med 2008; 177: 396-401.

45. Frei A, Siebeling L, Wolters C, et al. The inaccuracy of patient recall for COPD exacerbation rate estimation and its implications: results from central adjudication. Chest 2016; 150: 860-868.

46. Ejiofor SI, Stolk J, Fernandez P, et al. Patterns and characterization of COPD exacerbations using real-time data collection. Int J Chron Obstruct Pulmon Dis 2017; 12: 427-434.

47. Mackay AJ, Donaldson GC, Patel ARC, et al. Usefulness of the chronic obstructive pulmonary disease assessment test to evaluate severity of COPD exacerbations. Am J Respir Crit Care Med 2012; 185: 1218-1224.

48. Jones PW, Chen WH, Wilcox TK, et al. Characterizing and quantifying the symptomatic features of COPD exacerbations. Chest 2011; 139: 1388-1394.

49. Kessler R, Partridge MR, Miravitlles M, et al. Symptom variability in patients with severe COPD: a pan-European cross-sectional study. Eur Respir J 2011; 37: 264-272.

50. Celli B, Vestbo J, Jenkins CR, et al. Sex differences in mortality and clinical expressions of patients with chronic obstructive pulmonary disease the TORCH experience. Am J Respir Crit Care Med 2011; 183: 317-322.

51. Oshagbemi OA, Keene SJ, Driessen JHM, et al. Trends in moderate and severe exacerbations among COPD patients in the UK from 2005 to 2013. Respir Med 2018; 144: 1-6.

52. Woodruff PG, Barr RG, Bleecker E, et al. Clinical significance of symptoms in smokers with preserved pulmonary function. N EnglJ Med 2016; 374: 1811-1821.

53. Strassmann A, Frei A, Haile SR, et al. Commonly used patientreported outcomes do not improve prediction of COPD exacerbations: a multicenter $4 \frac{1}{2}$ year prospective cohort study. Chest 2017; 152: 1179-1187.

54. Mackay AJ, Donaldson GC, Patel AR, et al. Detection and severity grading of COPD exacerbations using the exacerbations of chronic pulmonary disease tool (EXACT). Eur Respir J 2014; 43: 735-744.

55. Effing TW, Kerstjens HAM, Monninkhof EM, et al. Definitions of exacerbations: does it really matter in clinical trials on COPD? Chest 2009; 136: 918-923.

56. Mackay AJ, Kostikas K, Murray L, et al. Patient-reported outcomes for the detection, quantification, and evaluation of chronic obstructive pulmonary disease exacerbations. Am J Respir Crit Care Med 2018; 198: 730-738.

57. Sanchez-Morillo D, Fernandez-Granero MA, Leon-Jimenez A. Use of predictive algorithms in-home monitoring of chronic obstructive pulmonary disease and asthma: a systematic review. Chron Respir Dis 2016; 13: 264-283.

58. Choi HS, Park YB, Shin KC, et al. Exacerbations of chronic obstructive pulmonary disease tool to assess the efficacy of acute treatment. Int J Chron Obstruct Pulmon Dis 2019; 14: 471-478.

59. Calverley P, Pauwels Dagger R, Lofdahl CG, et al. Relationship between respiratory symptoms and medical treatment in exacerbations of COPD. Eur Respir J 2005; 26: 406-413

60. Vestbo J, Leather D, Diar Bakerly N, et al. Effectiveness of fluticasone furoate-vilanterol for COPD in clinical practice. N EnglJ Med 2016; 375: 1253-1260.

61. Criner GJ, Connett JE, Aaron SD, et al. Simvastatin for the prevention of exacerbations in moderate-to-severe COPD. N EnglJ Med 2014; 370: 2201-2210.

62. Magnussen $\mathrm{H}$, Disse B, Rodriguez-Roisin R, et al. Withdrawal of inhaled glucocorticoids and exacerbations of COPD. N Engl J Med 2014; 371: 1285-1294.

63. Beeh KM, Glaab T, Stowasser S, et al. Characterisation of exacerbation risk and exacerbator phenotypes in the POETCOPD trial. Respir Res 2013; 14: 116.

64. Calverley PMA, Martinez FJ, Fabbri LM, et al. Does roflumilast decrease exacerbations in severe COPD patients not controlled by inhaled combination therapy? The REACT study protocol. Int J Chron Obstruct Pulmon Dis 2012; 7: 375-382.

65. Halpin DMG, Dransfield MT, Han MK, et al. The effect of exacerbation history on outcomes in the IMPACT trial. Eur Respir J 2020; 55: 1901921.

66. Burkes RM, Gassett AJ, Ceppe AS, et al. Rural residence and chronic obstructive pulmonary disease exacerbations. analysis of the SPIROMICS cohort. Ann Am Thorac Soc 2018: 15: 808-816.

67. Jenkins CR, Wen F-Q, Martin A, et al. The effect of lowdose corticosteroids and theophylline on the risk of acute exacerbations of COPD: the TASCS randomised controlled trial. Eur Respir J 2021; 57: 2003338.

68. Gadoury MA, Schwartzman K, Rouleau M, et al. Selfmanagement reduces both short- and long-term hospitalisation in COPD. Eur Respir J 2005; 26: 853-857.

69. Howcroft M, Walters EH, Wood-Baker R, et al. Action plans with brief patient education for exacerbations in chronic obstructive pulmonary disease. Cochrane Database Syst Rev 2016; 12: CD005074

70. Wedzicha JA, Banerji D, Chapman KR, et al. Indacaterolglycopyrronium versus salmeterol-fluticasone for COPD. N EnglJ Med 2016; 374: 2222-2234.

71. Calverley PMA, Anzueto AR, Carter K, et al. Tiotropium and olodaterol in the prevention of chronic obstructive pulmonary disease exacerbations (DYNAGITO): a doubleblind, randomised, parallel-group, active-controlled trial. Lancet Respir Med 2018; 6: 337-344.

72. Agusti A, Edwards LD, Rennard SI, et al. Persistent systemic inflammation is associated with poor clinical outcomes in COPD: a novel phenotype. PLoS One 2012; 7: e37483.

73. Celli B, Locantore N, Yates JC, et al. Markers of disease activity in COPD: an 8-year mortality study in the ECLIPSE cohort. Eur Respir J 2021; 57: 2001339.

74. Bafadhel M, Singh D, Jenkins C, et al. Reduced risk of clinically important deteriorations by ICS in COPD is eosinophil dependent: a pooled post-hoc analysis. Respir Res 2020; 21: 17.

75. Dickens JA, Miller BE, Edwards LD, et al. COPD association and repeatability of blood biomarkers in the ECLIPSE cohort. Respir Res 2011; 12: 146

76. Perera WR, Hurst JR, Wilkinson TM, et al. Inflammatory changes, recovery and recurrence at COPD exacerbation. Eur Respir J 2007; 29: 527-534.

77. Leidy NK, Wilcox TK, Jones PW, et al. Standardizing measurement of chronic obstructive pulmonary disease exacerbations. Reliability and validity of a patient-reported diary. Am J Respir Crit Care Med 2011; 183: 323-329.

78. Leidy NK, Murray LT. Patient-reported outcome (PRO) measures for clinical trials of COPD: the EXACT and E-RS. COPD 2013; 10: 393-398.

79. Global Initiative for Chronic Obstructive Lung Disease. 2021 Global Strategy for the Diagnosis, Management, and Prevention of Chronic Obstructive Pulmonary Disease. https://goldcopd.org/2021-gold-reports/

80. Aaron SD, Donaldson GC, Whitmore GA, et al. Time course and pattern of COPD exacerbation onset. Thorax 2012; 67: 238-243.

81. Trappenburg JC, van Deventer AC, Troosters T, et al. The impact of using different symptom-based exacerbation algorithms in patients with COPD. Eur Respir J 2011; 37: 1260-1268.

82. Ferguson GT, Skarby T, Nordenmark LH, et al. Unreported and overlooked: a post hoc analysis of COPD symptom-related attacks from the RISE study. Int J Chron Obstruct Pulmon Dis 2020; 15: 3123-3134

83. Barrecheguren M, Monteagudo M, Ferrer J, et al. Treatment patterns in COPD patients newly diagnosed in primary care. A population-based study. Respir Med 2016; 111: 47-53.

84. Beran D, Zar HJ, Perrin C, et al. Burden of asthma and chronic obstructive pulmonary disease and access to essential medicines in low-income and middle-income countries. Lancet Respir Med 2015; 3: 159-170.

85. Linnell J, Hurst JR. COPD Exacerbations: a patient and physician's perspective. Adv Ther 2020; 37: 10-16. 
86. Miravitlles M, Anzueto A, Legnani D, et al. Patient's perception of exacerbations of COPD - the PERCEIVE study. Respir Med 2007; 101: 453-460.

87. Rabe KF, Fabbri LM, Vogelmeier C, et al. Seasonal distribution of COPD exacerbations in the prevention of exacerbations with tiotropium in COPD trial. Chest 2013; 143: 711-719.

88. Mehanni S, Jha D, Kumar A, et al. Implementing a quality improvement initiative for the management of chronic obstructive pulmonary disease in rural Nepal. BMJ Open Qua 2019; 8: e000408.

89. Siddharthan T, Grigsby MR, Goodman D, et al. Association between household air pollution exposure and chronic obstructive pulmonary disease outcomes in 13 low- and middle-income country settings. Am J Respir Crit Care Med 2018; 197: 611-620.

90. Burney P. Chronic respiratory disease - the acceptable epidemic? Clin Med 2017; 17: 29-32.
91. Morgan BW, Grigsby MR, Siddharthan T, et al. Epidemiology and risk factors of asthma-chronic obstructive pulmonary disease overlap in low- and middle-income countries. J Allergy Clin Immunol 2019; 143: 1598-1606.

92. Berghaus TM, Karschnia P, Haberl S, et al. Disproportionate decline in admissions for exacerbated COPD during the COVID-19 pandemic. Respir Med 2021; in press [https:// doi.org/10.1016/j.rmed.2020.106120].

93. Avdeev S, Moiseev S, Brovko M, et al. Low prevalence of bronchial asthma and chronic obstructive lung disease among intensive care unit patients with COVID-19. Allergy 2020; 75 : 2703-2704.

94. Chan KPF, Ma TF, Kwok WC, et al. Significant reduction in hospital admissions for acute exacerbation of chronic obstructive pulmonary disease in Hong Kong during coronavirus disease 2019 pandemic. Respir Med 2020; 171: 106085 